\title{
TITLE:
}

\section{Chemogenetic dissection of the primate prefronto-subcortical pathways for working memory and decision-making}

\section{AUTHOR(S):}

Oyama, Kei; Hori, Yukiko; Nagai, Yuji; Miyakawa, Naohisa; Mimura, Koki; Hirabayashi, Toshiyuki; Inoue, Ken-ichi; ... Takada, Masahiko; Higuchi, Makoto; Minamimoto, Takafumi

\section{CITATION:}

Oyama, Kei ... [et al]. Chemogenetic dissection of the primate prefronto-subcortical pathways for working memory and decision-making. Science Advances 2021, 7(26): eabg4246.

\section{ISSUE DATE:}

2021-06

\section{URL:}

http://hdl.handle.net/2433/263918

\section{RIGHT:}

Copyright (c) 2021 The Authors, some rights reserved; exclusive licensee American Association for the Advancement of Science. No claim to original U.S. Government Works.; This is an open-access article distributed under the terms of the Creative Commons Attribution-NonCommercial license, which permits use, distribution, and reproduction in any medium, so long as the resultant use is not for commercial advantage and provided the original work is properly cited. 


\section{Chemogenetic dissection of the primate prefronto-subcortical pathways for working memory and decision-making}

\author{
Kei Oyama' ${ }^{1}$, Yukiko Hori ${ }^{1}$, Yuji Nagai ${ }^{1}$, Naohisa Miyakawa ${ }^{1}$, Koki Mimura', \\ Toshiyuki Hirabayashi ${ }^{1}$, Ken-ichi Inoue ${ }^{2,3}$, Tetsuya Suhara', Masahiko Takada ${ }^{2}$, \\ Makoto Higuchi ${ }^{1}$, Takafumi Minamimoto ${ }^{1 *}$
}

The primate prefrontal cortex (PFC) is situated at the core of higher brain functions via neural circuits such as those linking the caudate nucleus and mediodorsal thalamus. However, the distinctive roles of these prefronto-subcortical pathways remain elusive. Combining in vivo neuronal projection mapping with chemogenetic synaptic silencing, we reversibly dissected key pathways from dorsolateral part of the PFC (dIPFC) to the dorsal caudate (dCD) and lateral mediodorsal thalamus (MDI) individually in single monkeys. We found that silencing the bilateral dIPFC-MDI projections, but not the dIPFC-dCD projections, impaired performance in a spatial working memory task. Conversely, silencing the unilateral dIPFC-dCD projection, but not the unilateral dIPFC-MDI projection, altered preference in a decision-making task. These results revealed dissociable roles of the prefronto-subcortical pathways in working memory and decision-making, representing the technical advantage of imaging-guided pathway-selective chemogenetic manipulation for dissecting neural circuits underlying cognitive functions in primates.

\section{INTRODUCTION}

The primate prefrontal cortex (PFC), especially its dorsolateral part (dlPFC), is well known to serve as the center of higher-order executive functions; it is uniquely developed in primates and underlies their distinctive cognitive abilities (1). These functions, however, do not solely rely on dlPFC neurons but also on their cooperative interactions with subcortical structures, including the dorsal caudate nucleus (dCD) and lateral mediodorsal thalamus (MDl) (2-4). For example, working memory and decision-making - two fundamental functions that rely on the dlPFC-are impaired by lesions in either caudate nucleus (CD) or mediodorsal thalamus (MD) (5-8), although the debate continues $(9,10)$. Deficits in these cognitive functions have also been implicated in aberrant functional connectivity between PFC and CD/MD in patients with neurological, neuropsychiatric, or substance abuse disorders (11-13). Despite the growing evidence regarding the importance of dlPFC-derived cognitive signals in $\mathrm{dCD} / \mathrm{MDl}$, their causal contributions to working memory and decision-making remain unidentified in primates.

Anatomical studies have shown that the prefronto-subcortical projections arise from deep layers in monkeys (14-19). In addition, dlPFC, dCD, and/or MDl constitute two loop circuits, namely, the cortico-basal ganglia-thalamo-cortical loop and the cortico-thalamocortical loop, which partially overlap (18). Accordingly, this raises the critical open question of whether the dlPFC to $\mathrm{dCD} / \mathrm{MDl}$ pathways are involved in cognitive functions similarly or differently. To answer this question, it is essential to manipulate the prefronto-subcortical circuits independently.

Several studies have so far shown behavioral modification with reversible pathway-selective dissection in monkeys, where a doubleviral vector infection method was used to manipulate the activity of

\footnotetext{
'Department of Functional Brain Imaging, National Institutes for Quantum and Radiological Science and Technology, Chiba 263-8555 Japan. ${ }^{2}$ Systems Neuroscience Section, Primate Research Institute, Kyoto University, Inuyama, Aichi 484-8506, Japan. ${ }^{3}$ PRESTO, Japan Science and Technology Agency, Kawaguchi, Saitama, Japan. *Corresponding author. Email: minamimoto.takafumi@qst.go.jp
}

a single neuronal pathway (20-23). This approach, however, is not suitable for investigating multiple neural pathways independently within a single animal. Optogenetics is another approach to manipulating the neuronal activity of a specific pathway by optical stimulation of axon-terminal sites. Although a few studies have succeeded in pathway-specific optogenetic activation in nonhuman primates (24-26), optogenetic silencing of synaptic transmission remains challenging $(27,28)$. Moreover, these approaches require precise identification of the locations of anatomically connected multiple regions, i.e., viral injection or allocation of optic fiber, which is technically demanding when using nonhuman primates because of limited resources.

To elucidate the causal roles of distinct prefronto-subcortical pathways in macaque monkeys, in other words, the pathways from dlPFC to dCD (dlPFC-dCD) and from dlPFC to MDl (dlPFC-MDl), we applied one of the chemogenetic tools, designer receptors exclusively activated by designer drugs (DREADDs). While neuronal silencing can be achieved by activating an inhibitory DREADD (hM4Di) following systemic agonist delivery (29), activating hM4Di expressed at axon terminals through local agonist infusion also leads to suppression of synaptic transmission (30-34). Here, we developed imaging-guided chemogenetic synaptic silencing-a methodology for silencing neural pathways selectively with an agonist infused at hM4Di-positive dlPFC projection sites that are mapped in vivo beforehand by using positron emission tomography (PET) (35). Using this technique, we sequentially disconnected the bilateral/ unilateral dlPFC-dCD and dIPFC-MDl pathways reversibly to investigate the causal roles in working memory and decision-making, which we hypothesized as being distinctively governed by the two prefronto-subcortical pathways.

\section{RESULTS}

\section{PET visualizes hM4Di-positive terminal sites in dCD} and $M D I$ in vivo

We tested two monkeys in which hM4Di had been introduced into the bilateral dIPFC around the principal sulcus (Brodmann's area 46) 
through adeno-associated virus (AAV) vector injections (Fig. 1A). Several weeks after the injections, we performed PET imaging with a radiolabeled form of the highly potent DREADD-specific agonist, deschloroclozapine (DCZ) (35), to visualize hM4Di expression noninvasively. Increased $\left[{ }^{11} \mathrm{C}\right] \mathrm{DCZ}$ binding was localized not only in regions of the bilateral dlPFC (Fig. 1B) but was also visible as spots with a diameter of 3 to $5 \mathrm{~mm}$ in the dCD head and MDl (Fig. 1, C and D). These PET signals reflected hM4Di expression at both the soma/dendrite and the axon terminal sites, confirmed by immunohistochemistry in Mk\#1 (fig. S1) and another monkey (Mk\#3) that received injections of an AAV vector encoding a fluorescent marker [monomergic Kusabira Orange $(\mathrm{mKO})$ ] similarly into the dIPFC (Fig. 1, E to J). In addition, the PET signal reflected hM4Di expression in the putamen but was not detectable in other projection sites (fig. S2). Thus, we noninvasively identified the target sites for local agonist infusion to reversibly disconnect either the dlPFC-dCD or dlPFC-MDl pathway individually.

\section{Chemogenetic silencing of the bilateral dIPFC-MDI pathways selectively impaired the performance of a delayed response task}

We then explored the contribution of the dIPFC-subcortical pathways to working memory function in the monkeys who performed a spatial delayed response task (Fig. 2A). Before pathway-selective silencing, we verified the effect of chemogenetic silencing of the bilateral dIPFC. Consistent with our previous findings (35), systemic delivery of a low dose of DCZ ( $100 \mu \mathrm{g} / \mathrm{kg}$, i.m.) drastically impaired the task performance in a delay-dependent manner, indicating that chemogenetic silencing of the bilateral dlPFC caused working memory deficits (Fig. 2, B and E). Subsequently, we attempted to silence the dIPFC terminals through microinfusion of DCZ solution into the hM4Di-positive regions of either dCD or MDl bilaterally (Fig. 2, $\mathrm{C}$ and $\mathrm{D}$, top). Infusion cannulae were placed under the guidance of computed tomography (CT) images overlaying PET and magnetic resonance (MR) images (Fig. 2, C and D, bottom). We adopted $100 \mathrm{nM}$ DCZ solution for microinfusion, because (i) preceding studies have used $\mathrm{CNO}$ (clozapine- $\mathrm{N}$-oxide, the most frequently used DREADD agonist) for local administration at $3 \mu \mathrm{M}$ to $1 \mathrm{mM}$ concentration $(30,31)$, which corresponds to $30 \mathrm{nM}$ to $100 \mu \mathrm{M} \mathrm{DCZ}$ in terms of its 100 -fold higher agonist potency for hM4Di, and (ii) $100 \mathrm{nM} \mathrm{DCZ}$ is low enough to avoid off-target effects considering its affinities for numerous central nervous system receptors or transporters (35). In both monkeys, local DCZ infusion into MDl prominently impaired the task performance, as compared to the control vehicle infusion (Fig. 2G). Impaired behavior in $\mathrm{MDl}$ infusions was consistently observed within a single testing session (30 to 90 min after infusion; fig. S3) and across the sessions [three-way analysis of variance (ANOVA); treatment $\times$ delay $\times$ session, main effect of session; $F_{(4,30)}=1.7, P=0.17$; using the same dataset as Fig. 2G, data of two monkeys were pooled]. This impairment also depended on the delay duration (Fig. 2G), indicating that the dlPFC-MDl pathway is critical for working memory function. By contrast, DCZ infusion into dCD had no significant effect on the task performance (Fig. 2F).

\section{Chemogenetic silencing of the unilateral dIPFC-dCD pathway altered the choice preference in a delayed response task}

Having demonstrated effective bilateral pathway-specific silencing, we next examined the effects of unilateral silencing of each pathway
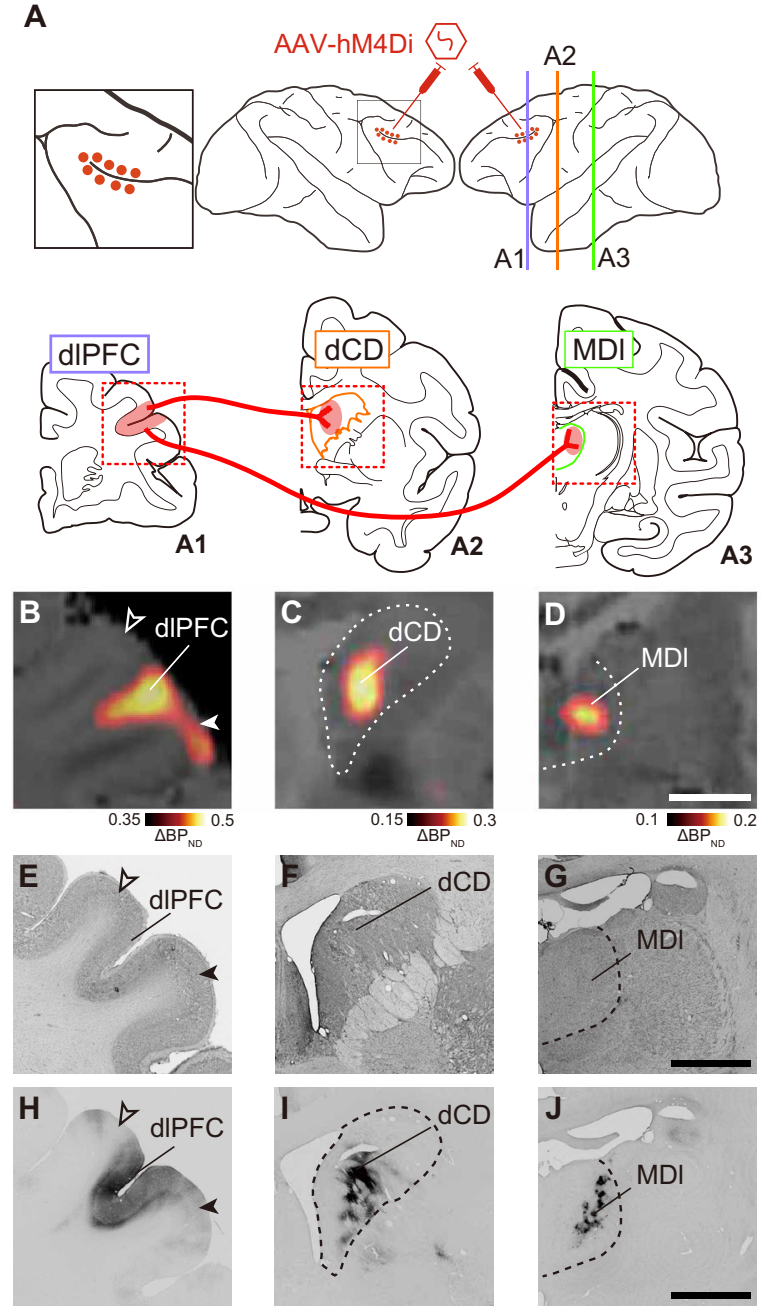

Fig. 1. PET visualizes hM4Di-positive terminal sites in dCD and MDI in vivo. (A) Illustration representing the target pathways for chemogenetic silencing. Top: Lateral view of injection sites. The left panel represents a zoomed-in view of the framed area of the center panel. Bottom: Coronal planes corresponding to the lines in the lateral view. Red dots (top) and shaded area (bottom) represent intended injection sites (dIPFC) of an AAV vector expressing hM4Di and its projections sites ( $\mathrm{dCD}$ and $\mathrm{MDI}$ ), while red wires represent projections from dIPFC to $\mathrm{dCD}$ and $\mathrm{MDI}$. (B to $D$ ) In vivo visualization of $h M 4 D i$ expression in dIPFC (B), dCD (C), and MDI (D). Coronal PET image showing specific binding of $\left[{ }^{11} \mathrm{C}\right] \mathrm{DCZ}$ subtracted before, from after the introduction of hM4Di, overlaying MR image of Mk\#2. (E to G) Corresponding Nissl-stained sections in dIPFC (E), dCD (F), and MDI (G), respectively. (H to J) Corresponding diaminobenzidine (DAB)-stained sections representing immunoreactivity against a reporter protein $(\mathrm{mKO})$ in $\operatorname{dIPFC}(\mathrm{H}), \mathrm{dCD}(\mathrm{I})$, and $\mathrm{MDI}(\mathrm{J})$ of a reference monkey that received viral vector injections into PFC in a similar manner. Dashed lines represent the borders of the caudate nucleus and mediodorsal thalamus, respectively. Open and filled arrowheads represent the dorsal and ventral borders of the target regions, respectively. Scale bars, $5 \mathrm{~mm}$. Regional binding potential relative to nondisplaceable radioligand, $\mathrm{BP}_{\mathrm{ND}}$.

on the task in the same animals (Fig. 3A). We expected that unilateral silencing would cause an imbalance in the information flow, thereby resulting in asymmetric behavioral changes between hemifields. We infused DCZ solution into either dCD or MDl (Fig. 3, B and C) (3 and $2 \mu \mathrm{l}$, respectively), which would not diffuse beyond the midline, warranting unilateral pathway-specific silencing (see Materials and Methods). 


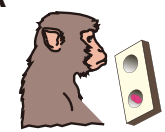

Cue

B

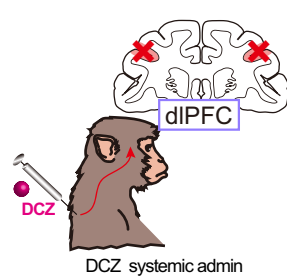

E
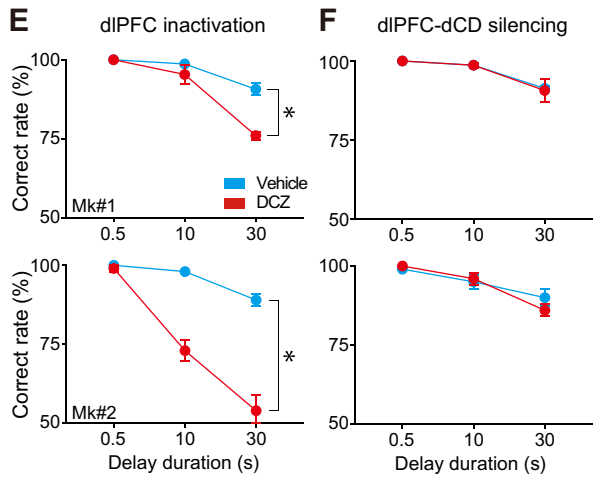

F dIPFC-dCD silencing

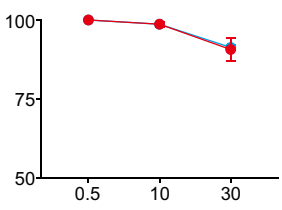

D
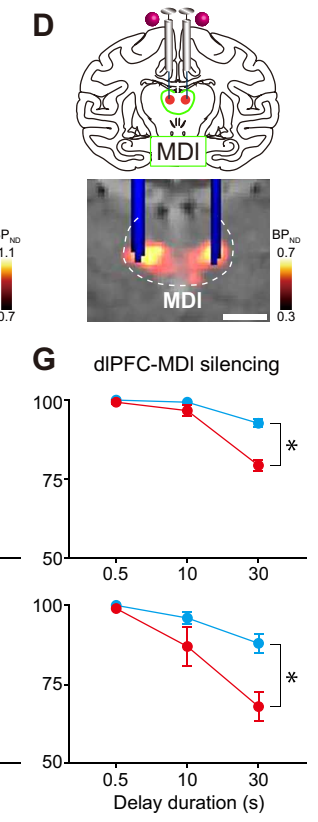

Fig. 2. Chemogenetic silencing of the bilateral dIPFC-MDI pathway selectively impaired the performance of a delayed response task. (A) Delayed response task. (B) Schematic diagram representing inactivation of dIPFC by systemic DCZ administration. (C) Chemogenetic silencing of the dIPFC-dCD pathway by local DCZ infusion ( $100 \mathrm{nM}, 3 \mu \mathrm{l}$ per site) into bilateral $\mathrm{dCD}$, specifically at $\mathrm{hM} 4 \mathrm{Di}$-positive dIPFC terminals sites. A CT image showing the infusion cannulae (blue) overlaying a structural MR image (gray), and a PET image showing a high $\left[{ }^{11} \mathrm{C}\right] \mathrm{DCZ}$ binding region (hM4Di expression, hot color). (D) Chemogenetic silencing of the dIPFC-MDI pathway by local DCZ infusion ( $2 \mu \mathrm{l}$ per site) into bilateral MDI. (E) Behavioral effects of chemogenetic dIPFC inactivation. Correct performance rate (means \pm SEM, $n=5$ sessions for each treatment in each monkey) following DCZ (red) and vehicle infusions (cyan) are shown for two monkeys [two-way ANOVA with treatment $x$ delay; treatment: $\mathrm{Mk} \# 1, F_{(1,24)}=20.8, P<0.001 ; \mathrm{Mk} \# 2, F_{(1,24)}=90.8, P<0.001$; delay: Mk\#1, $F_{(2,24)}=60.9, P<0.001 ;$ Mk\#2, $F_{(2,24)}=57.4, P<0.001$; interaction: Mk\#1, $F_{(2,24)}=$ $\left.11.4, P<0.001 ; M k \# 2, F_{(2,24)}=22.3, P<0.001\right]$. (F) Same as (E) but for the PFC-CD pathway [treatment: Mk\#1, $F_{(1,24)}=0.03, P=0.86 ; \mathrm{Mk} \# 2, F_{(1,24)}=0.20, P=0.66$; delay: Mk\#1, $F_{(2,24)}=19.3, P<0.001 ;$ Mk\#2, $F_{(2,24)}=20.0, P<0.001$; interaction: Mk\#1, $F_{(2,24)}=$ $\left.0.03, P=0.97 ; \mathrm{Mk} 22, F_{(2,24)}=1.2, P=0.31\right]$. (G) Same as (E) but for the PFC-MD pathway [treatment: Mk\#1, $F_{(1,24)}=32.9, P<0.001$; MK\#2, $F_{(1,24)}=12.6, P=0.002$; delay: Mk\#1, $F_{(2,24)}=79.0, P<0.001 ; M k \# 2, F_{(2,24)}=19.8, P<0.001$; interaction: Mk\#1, $\left.F_{(2,24)}=16.5, P<0.001 ; M k \# 2, F_{(2,24)}=3.8, P=0.04\right]$. Dashed lines represent the borders of the caudate nucleus and mediodorsal thalamus, respectively. Asterisks indicate $P<0.05$ for significant main effect of treatment. Scale bars, $5 \mathrm{~mm}$.

In contrast to the bilateral silencing, local DCZ infusion into unilateral $\mathrm{MDl}$ did not affect the performance of delayed response task in either monkey (Fig. 3E), suggesting that the intact function of either dlPFC$\mathrm{MDl}$ pathway is sufficient for cognitive behavior as was suggested by previous lesion studies in monkeys (36). Local DCZ infusion into unilateral $\mathrm{dCD}$, on the other hand, significantly decreased the correct performance rate in trials with food given in the contralateral well to the injected side (Fig. 3D, right) and tended to improve the performance on the ipsilateral side (Fig. 3D, left). Given that the bilateral silencing spared working memory function, it was unlikely to be due to a loss of working memory. Together, these results suggest that the dIPFC-MDl pathway, but not the dlPFC-dCD pathway, plays an essential role in working memory function, where both sides of the pathways can complement each other.

\section{Chemogenetic silencing of the dIPFC-dCD pathway induced laterality bias in a free-choice task}

As demonstrated above, DCZ infusion into unilateral dCD led to hemifield-dependent behavioral changes in a working memory performance, while the bilateral injections did not. These observations led us to postulate that unilateral silencing of the dIPFC-dCD pathway induces laterality bias in decision-making in a hemifield-dependent fashion as suggested in previous single-unit recording and lesion studies showing an involvement of dCD in such a phenomenon (37). To identify the contribution of the dlPFC-dCD/MDl pathways in decision-making-another function being responsible for the prefronto-subcortical network, we focused on a behavioral bias under a free-choice situation. We used a free-choice task, in which the monkeys were allowed to pick up food from either left or right baited wells without instructions (Fig. 4A). If signals processed via the dIPFC-dCD pathway promoted contralateral choices, silencing of this pathway on one side of the brain would increase a choice of wells on the same side. As we predicted, unilateral DCZ infusion into CD (Fig. 4B) significantly increased the choice of ipsilateral wells compared with the vehicle infusion, indicating that ipsilateral bias emerged (Fig. 4D). This impairment was not due to the inability of voluntary attention to the contra-hemifield (i.e., contralateral hemineglect) as seen in the case of unilateral dopamine depletion from dCD (38), because the animals maintained normal spontaneous eye movements by viewing both the ipsi- and contra-hemifields at the same frequency (fig. S4). Unilateral DCZ infusion into MDl (Fig. 4C), on the other hand, did not change the tendency of the monkeys' choices (Fig. 4E). Thus, the overall results suggest that the dlPFC-dCD pathway in each hemisphere may contribute to decisionmaking by biasing one's action toward the contralateral side without direct involvement of working memory or oculomotor function.

Last, we verified that any behavioral side effects were not caused by the effect of DCZ infusion into nontarget sites, i.e., areas without innervations of hM4Di-positive axons. We infused DCZ into brain regions where discernible hM4Di expression was undetected, including the ventral putamen and ventroanterior thalamus, the structures located adjacent to $\mathrm{dCD}$ and $\mathrm{MDl}$, respectively. In these cases, the monkeys displayed no discernible behavioral changes in either task (fig. S5), thus supporting that our data described above are attributable to the chemogenetic silencing of hM4Di-positive dlPFC-derived axon terminals.

\section{DISCUSSION}

Here, we chemogenetically dissected the neuronal information flow from the dlPFC to two subcortical areas, MDl and dCD, in individual monkeys. Our results provided direct evidence for a functional dissociation between PFC-MDl and PFC-dCD pathways in the performance of the delayed response task and free-choice task. We also showed that bilateral and unilateral silencing had different impacts on behavior, with their effects being consistent across tasks. Given that the dIPFC is unique to primates and has no clear homolog in other species such as rodents (1), our results would provide valuable 
A

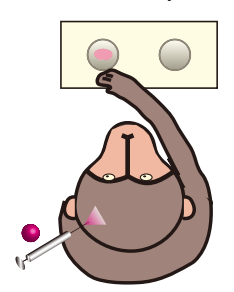

Ipsilateral trial

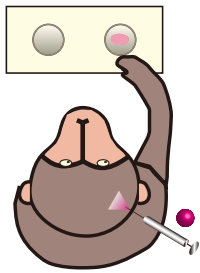

Contralateral trial

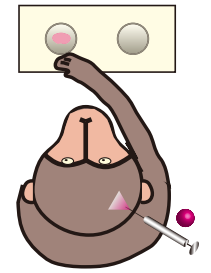

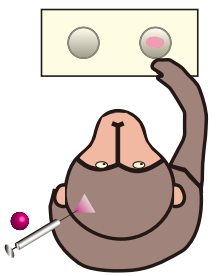

B

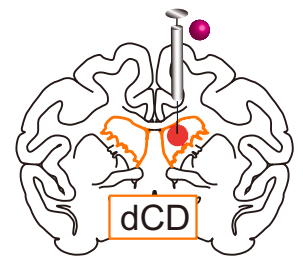

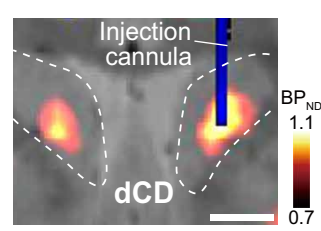

dIPFC-dCD silencing

D
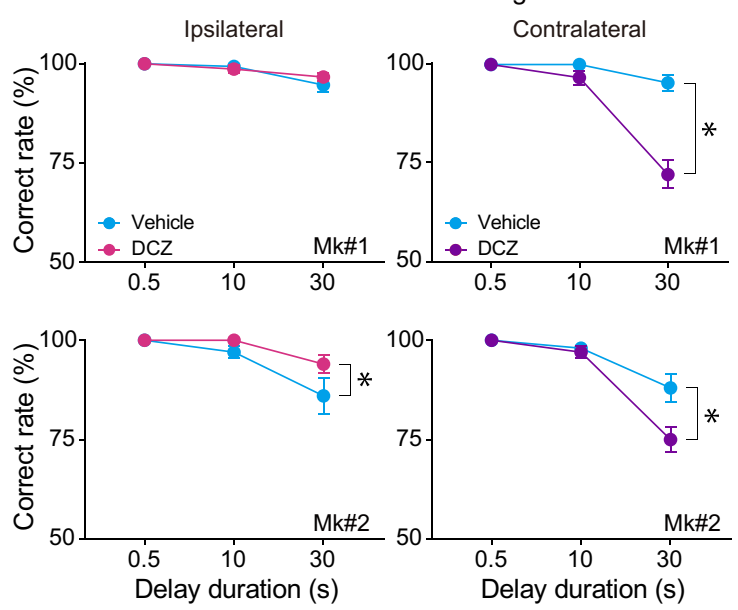

C
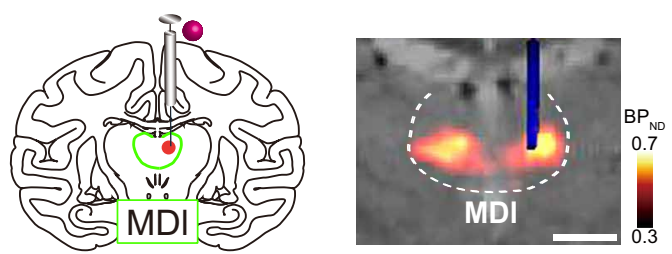

$E$
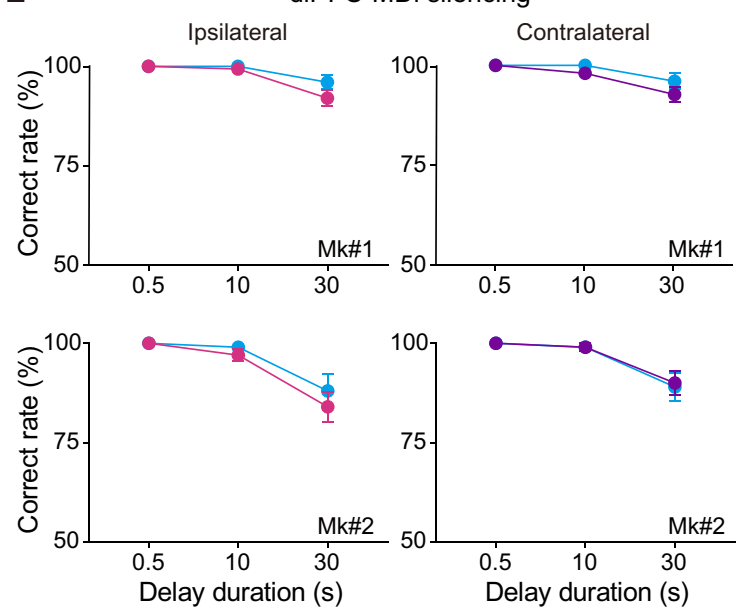

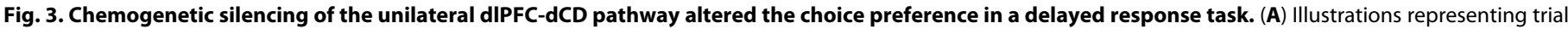

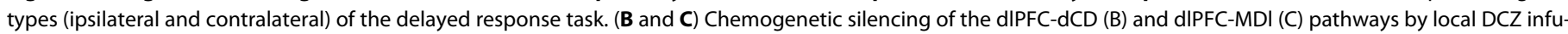

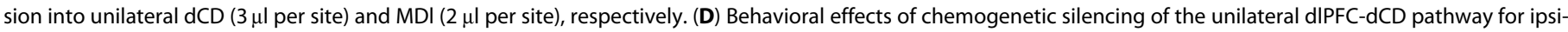

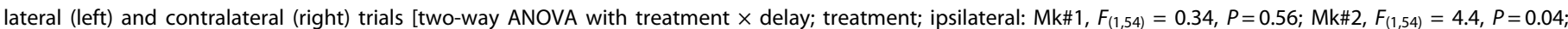

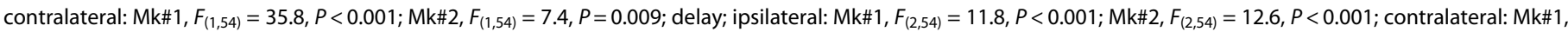

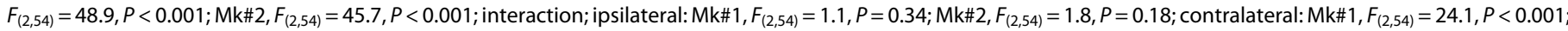

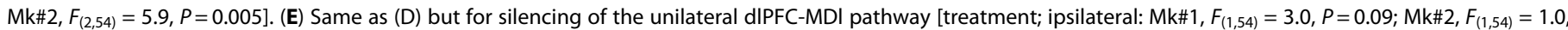

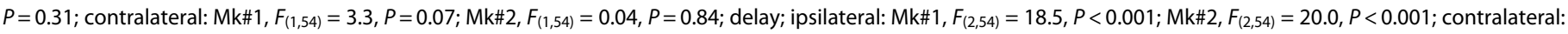

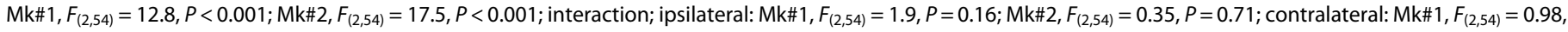

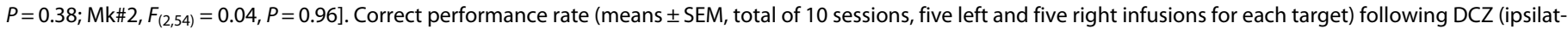
eral, magenta; contralateral, purple) and vehicle infusions (cyan) are shown for two monkeys. Arrangements are the same as in Fig. 2.

insights into the neural basis for higher-order executive functions of primates.

Anatomically, dlPFC and dCD/MDl constitute two loop circuits, namely, the cortico-basal ganglia-thalamo-cortical and the corticothalamo-cortical loop. The former is a part of a series of parallelprojecting, largely segregated circuits that arise from the cortex and link the striatum (including dCD), pallidum, and thalamus back to the same original cortical area $(18,19)$. The latter is a reciprocal connection between the cortex and thalamus $(39,40)$. Thus, dlPFC communicates with two partially overlapped subcortical circuits, where MDl receives information from the same dlPFC area both directly and indirectly. Unlike conventional inactivation or lesion experiments, the chemogenetic manipulation described here dissected the dlPFC to the $\mathrm{dCD} / \mathrm{MDl}$ pathways independently, thereby allowing us to untangle the direct and indirect effects on MDl. Our findings suggest that the dlPFC-subcortical projections originating in the same region play distinct roles in cognitive functions. What is the mechanistic explanation for the differential contribution? We speculate on the following possibilities. First, the two pathways might have originated from distinct neuronal populations that distinctly signal critical information for working memory or decisionmaking. It has been shown that the dlPFC-MDl and dlPFC-dCD projections arise from deep layers $(14,15)$, while a small proportion of dlPFC neurons have axonal branches that innervate both sites (41). Thus, there might exist anatomically intermingled subpopulations in the dlPFC. Second, the two pathways might convey similar 
A

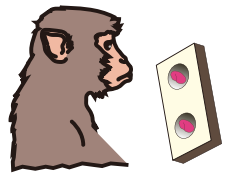

Cue

B

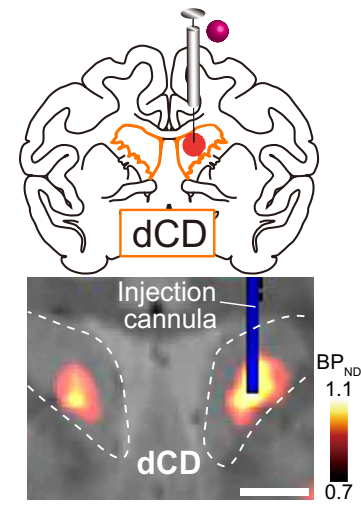

D

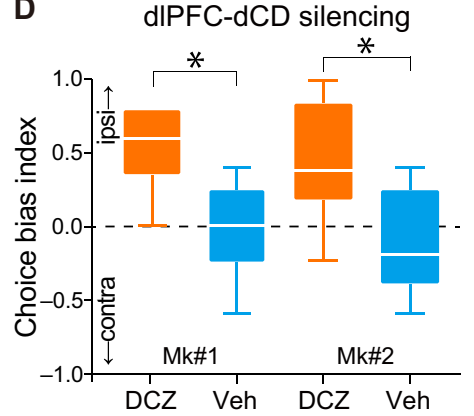

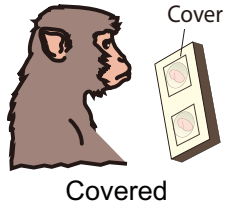

C

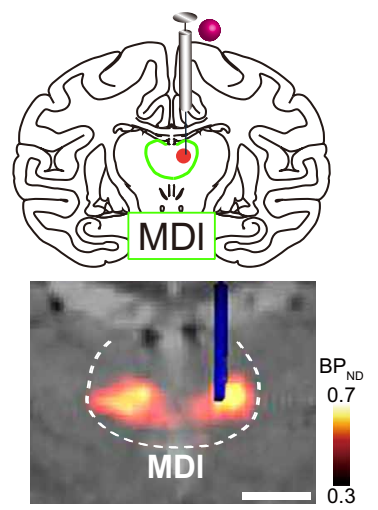

E

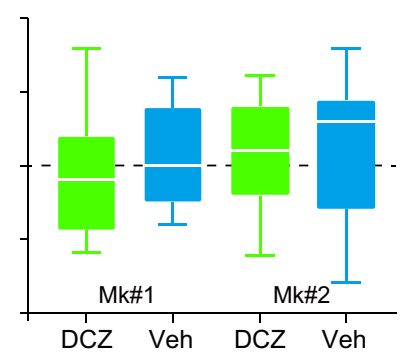

Fig. 4. Chemogenetic silencing of the unilateral dIPFC-dCD pathway induced laterality bias in a free-choice task. (A) Free-choice task. (B and C) Chemogenetic unilateral silencing of the dIPFC-dCD (B) and dIPFC-MDI pathways (C) by local unilateral DCZ infusion. Arrangements are the same as in Fig. 2 (C and D). (D and E) Choice bias index of free-choice task following unilateral $D C Z$ infusion into $d C D(D$, orange; Brunner-Munzel test: $\mathrm{Mk} \# 1, \mathrm{BM}$ value $=-6.7, P<0.001 ; \mathrm{Mk} \# 2, \mathrm{BM}$ value $=-5.0$, $P<0.001)$ and $\mathrm{MDI}(\mathrm{E}$, green; $\mathrm{Mk \# 1,BM}$ value $=0.79, P=0.44 ; \mathrm{Mk} \# 2, \mathrm{BM}$ value $=0.37$, $P=0.72$ ) and control vehicle infusions (cyan). Boxplots indicate the median (line within the box), the first to third quartiles (box), and the largest and smallest data points (whiskers) (total of 10 sessions, five left and five right infusions for each target). Asterisks indicate $P<0.05$ for significant difference. Scale bars: $5 \mathrm{~mm}$.

information, which is processed differently in each subcortical area, leading to the distinct functions. Further study would be required to clarify the underlying mechanism of the causal role of the two pathways. Third, information from the dlPFC-dCD pathway might be conveyed to another output nucleus, such as superior colliculus, to engage in decision-making (42), without direct involvement in spontaneous saccades. Whatever the mechanism is, our results indicate that a single dIPFC region exerts different cognitive functions depending on which subcortical area it interacts with. These pathwayspecific functions have been overlooked, because they cannot be distinguished in conventional neuronal recording or inactivation experiments. The results of these experiments have demonstrated functional localization in multiple PFC areas (43). Our results extend this view and highlight the functional heterogeneity of PFC-subcortical circuits in higher-order executive functions.

The importance of dIPFC and MDl in implementing working memory has been repeatedly shown (44). A human imaging study reported that the strength of functional connectivity between dlPFC and $\mathrm{MDl}$ during a working memory task correlated with memory load (13), suggesting their cooperative involvement. In a monkey study, similar proportions of neurons in $\mathrm{dlPFC}$ and $\mathrm{MDl}$ were active during the delay period in working memory task, whereas a greater proportion of neurons in $\mathrm{MDl}$ than $\mathrm{dlPFC}$ participated in the response phase, suggesting that the PFC-MD pathway is involved in both maintenance and transformation of working memory (45). Our findings corroborated these notions by providing direct evidence for the causal involvement of the primate dlPFC-MDl pathway in working memory. Our results also extend the recent findings in a rodent study that showed an essential role of the dlPFC-MDl pathway in a spatial working memory task (7) and together imply possible common neural substrates between primates and rodents. It should also be noted that the involvement of $\mathrm{MDl}$ in working memory remains controversial, as there are several reports showing no working memory deficits associated with MD lesions $(46,47)$, and silencing of PFC-MD and/or MD-PFC pathways impaired other cognitive functions regardless of sensory modality $(48,49)$.

Silencing of the unilateral dIPFC-MDl pathway did not have an impact on the behavioral performance of the delayed response task. This result suggests that the unilateral dysfunction of this pathway would be compensated by the other, which contrasted with the case in which the unilateral dysfunction of dIPFC per se that has been reported to cause deficits in contra-hemifield working memory (50). This finding also raised the possibility that the dlPFC-MDl pathway would convey nonlateralized information in terms of visuospatial processing, at least in relation to implementing working memory, which was supported by a previous electrophysiological recording in monkey $\mathrm{MDl}$ (51). Future studies that combine electrophysiological recording and pathway-specific silencing would be required to reveal the essential information flow from dlPFC to MDl.

On the other hand, our findings also suggest that the dlPFC-dCD pathway does not play an essential role in the performance of a spatial delayed response task, which is one of the major paradigms when examining spatial working memory $(52,53)$. However, this does not necessarily mean that the dlPFC-dCD pathway is irrelevant to working memory. It has been proposed that PFC-striatal interactions act as a gate for selecting the contents of working memory that should be stored via the prefronto-basal ganglia-thalamus network $(54,55)$. For example, functional connectivity between the dorsolateral part of the PFC to the anterior striatum in humans (presumably homologous to dlPFC-dCD in monkeys) was modulated by the novelty of visual cues to be memorized (56). In our task setting, monkeys were just required to memorize the location of the familiar foods, which might not require contribution of the dlPFC-dCD pathway due to its simplicity. Together, our results show that the dlPFC-dCD pathway might not play a pivotal role in implementing working memory, but they do not exclude the possibility that this pathway contributes to supporting working memory in a more complicated situation.

Another key finding of our study is that unilateral silencing of the dlPFC-dCD pathway resulted in biasing choices away from the contralateral option under the free-choice situation (Fig. 4). Unlike the visual hemineglect induced by unilateral dopamine depletion in $\mathrm{CD}(38,57)$, we did not find any significant deviation of 
spontaneous eye position (fig. S4). Thus the deficits observed here were not directly caused by alteration of eye movement or involuntary attention. Similar ipsiversive bias in a free-choice task has been reported to occur after unilateral deactivation of the dlPFC in monkeys (58). It has been proposed that the pathway from the PFC to $\mathrm{CD}$ conveys cognitive information while $\mathrm{CD}$ neurons represent specific actions/stimuli associated with value preferentially in the contralateral hemisphere to guide goal-directed behavior $(59,60)$. We speculate that unilateral silencing of the dlPFC-dCD pathway may disrupt the contra-preferred value representation in $\mathrm{dCD}$, leading to an increase in ipsilateral choices. This also explains our paradoxical result that unilateral, but not bilateral, dlPFC-dCD silencing produced behavioral bias in the delayed response task. Contrary to the dlPFC-dCD pathway, unilateral silencing of the dlPFC-MDl pathway did not alter the performance of the free-choice task. Because the primate $\mathrm{MDl}$ is known to be involved in adaptive decision-making, such as value-based choice behavior (61), our results are possibly attributable to compensation by the intact side of the dIPFC-MDl pathway that may process nonlateralized information, as discussed above.

Recent advances in specific neuronal manipulation tools have drastically enhanced our understanding of the functional roles of individual neural pathways in small animals such as rodents $(29,62)$. Nonetheless, the application of pathway-selective manipulations to nonhuman primates is still largely limited by the use of either optogenetic terminal activation (24-26) or double-vector methods (20-23), neither of which is capable of testing dissociable function of multiple neural pathways in individual subjects. Using a chemogenetic approach, the current study succeeded in the double dissociation of the roles of two distinct pathways in individual monkeys. Our findings were largely owing to highly reproducible manipulations of a specific pathway, which was achieved by in vivo projection mapping of the hM4Di-positive sites in $\mathrm{dCD} / \mathrm{MDl}$ and accurate positioning of the injection cannulae into the areas proven by CT imaging following every injection. Imaging-guided chemogenetic synaptic silencing permits us to identify and dissect predefined circuits. In combination with methods to monitor neuronal activity, such as electrophysiological recording or functional neuroimaging, it would be possible to specify the precise content of signals processed via the prefronto-subcortical network. Given the accurate targeting with applicable capability, imaging-guided chemogenetics affords powerful and unique strategies for pathway-selective dissection, thereby expanding our opportunities of linking neural circuits to behaviors in primates.

In summary, the present study has demonstrated a dissociation of the cognitive roles that the two dlPFC pathways toward $\mathrm{MDl}$ and $\mathrm{dCD}$ exert in working memory and decision-making, respectively. Given the anatomical and functional heterogeneity of the primate PFC, our findings provide invaluable insights toward the understanding of a neural basis for higher-order executive functions/ dysfunctions associated with neuropsychiatric disorders.

\section{MATERIALS AND METHODS \\ Subjects}

All experimental procedures involving animals were carried out in accordance with the Guide for the Care and Use of Laboratory Animals (National Research Council of the U.S. National Academy of Sciences) and Institutional Animal Care and Use Committee and were approved by the Animal Ethics Committee of the National
Institutes for Quantum and Radiological Science and Technology. Three macaque monkeys [Mk\#1: male Rhesus (Macaca mulatta), Mk\#2: female Japanese monkey (Macaca fuscata), and Mk\#3: female Rhesus (M. mulatta); 5.0, 5.7, and $4.2 \mathrm{~kg}$; ages 5, 6, and 9 years at the beginning of experiments] were used. The monkeys were kept in individual primate cages in an air-conditioned room. A standard diet, supplementary fruits/vegetables, and a tablet of vitamin C (200 mg) were provided daily.

\section{Viral vector production}

AAV1 (AAV1-hSyn-hM4Di-IRES-AcGFP) and AAV2 (AAV2-CMV$\mathrm{mKO}$ ) vectors were produced by helper-free triple transfection procedure, which was purified by affinity chromatography (GE Healthcare, Chicago, USA). Viral titer was determined by quantitative polymerase chain reaction using Taq-Man technology (Life Technologies, Waltham, USA).

\section{Surgical procedures and viral vector injections}

Surgeries were performed under aseptic conditions in a fully equipped operating suite. We monitored body temperature, heart rate, $\mathrm{SpO}_{2}$, and tidal $\mathrm{CO}_{2}$ throughout all surgical procedures. Monkeys were immobilized by intramuscular injection of ketamine (5 to $10 \mathrm{mg} / \mathrm{kg}$ ) and xylazine $(0.2$ to $0.5 \mathrm{mg} / \mathrm{kg}$ ) and intubated with an endotracheal tube. Anesthesia was maintained with isoflurane (1 to $3 \%$, to effect). Before surgery, MR imaging (7 tesla $400 \mathrm{~mm} / \mathrm{SS}$ system, NIRS/ KOBELCO/Brucker) and X-ray CT scans (Accuitomo170, J. MORITA CO., Kyoto, Japan) were performed under anesthesia (continuous infusion of propofol 0.2 to $0.6 \mathrm{mg} / \mathrm{kg}$ per min, intravenously). Overlaid MR and CT images were created using PMOD image analysis software (PMOD Technologies Ltd., Zurich, Switzerland) to estimate stereotaxic coordinates of target brain structures.

Two monkeys (Mks\#1 and \#2) were injected AAV1-hSyn-hM4DiIRES-AcGFP $\left(4.7 \times 10^{13}\right.$ particles $\left./ \mathrm{ml}\right)$ into the bilateral prefrontal cortex (Brodmann's area 46), and one monkey (Mk\#3) was injected AAV2-CMV-mKO $\left(1.7 \times 10^{13}\right.$ particles $\left./ \mathrm{ml}\right)$ into the unilateral prefrontal cortex. After retracting skin and galea, the frontal cortex was exposed by removing a bone flap and reflecting the dura mater. Handheld injections were made under visual guidance through an operating microscope (Leica M220, Leica Microsystems GmbH, Wetzlar, Germany), with care taken to place the beveled tip of the Hamilton syringe containing the viral vector at an oblique angle to the brain surface. The needle was inserted into the intended area of injection by one person, and a second person pressed the plunger to expel approximately $1 \mu \mathrm{l}$. Nine tracks were injected in each hemisphere; one was located $1 \mathrm{~mm}$ posterior to the caudal tip of the principal sulcus, and the others were located along the dorsal (four tracks) and ventral (four tracks) bank of the principal sulcus posterior to the rostral tip of the ascending limb of the arcuate sulcus (Fig. 1). Viral vectors were injected 3 to $5 \mu \mathrm{l}$ per track depending on the depth. Totals of 35 and $37 \mu \mathrm{l}$ for Mk\#1 and 40 and $44 \mu \mathrm{l}$ for Mk\#2 of viral aliquots were injected into the right and left hemispheres, respectively, and $20 \mu \mathrm{l}$ into the left hemisphere of $\mathrm{Mk} \# 3$.

\section{Behavioral task}

Two monkeys (Mks\#1 and \#2) were tested with a spatial delayed response task (Fig. 2). The protocol was almost the same as that of our previous study (35). Behavioral testing was conducted in a sound-attenuated room. Monkeys were seated in a monkey chair from which they could reach out one hand and take food to their 
mouths. A wooden table with two food wells was placed in front of the monkeys, and a screen was placed between the monkeys and the table. First, a piece of food reward (raisin or snack) was placed in one of the two food wells, and then both wells were covered with wooden plates. Then, the screen was placed for $0.5,10$, or $30 \mathrm{~s}$, which served as delay periods. The position of the baited well (left or right) and the delay period $(0.5,10$, or $30 \mathrm{~s})$ were determined pseudo-randomly. After the delay period, the screen was removed, and the monkeys were allowed to select either food well to get the food. The monkeys were allowed to get the food if they reached for the correct food well and removed the cover plate. The intertrial interval was set at $10 \mathrm{~s}$. A daily session lasted about 1 hour, consisting of three blocks of 30 trials (10 trials for each delay of $0.5,10$, or $30 \mathrm{~s}$ ) for Mk\#1 and two blocks of 30 trials for Mk\#2. Each of the monkeys performed the single sessions individually. The blocks were separated by 5 -min rest periods. The task was tested under both bilateral and unilateral silencing conditions.

Two monkeys (Mks\#1 and \#2) were also tested with a free-choice task only under unilateral silencing conditions (Fig. 4). In this task, both wells were baited, and then the food wells were covered. Then, the table was placed in front of the monkeys and they were allowed to get the food. After the monkeys picked up one side of food, the food table was withdrawn, and then both wells were baited again for the next trial. The task consisted of 10 trials. The delayed response task and the free-choice task were conducted in the same sessions in a counterbalanced sequence.

\section{Drug administration}

DCZ (MedChemExpress) was dissolved in 2.5\% dimethyl sulfoxide (DMSO; FUJIFILM Wako Pure Chemical Co.). For systemic intramuscular injection, this stock solution was diluted in saline to a final volume of $0.1 \mathrm{mg} / \mathrm{kg}$ and injected intramuscularly $15 \mathrm{~min}$ before the beginning of the experiments. Note that DCZ itself did not affect the performance in monkeys without hM4Di expression $(35,63)$. For microinfusion, DCZ was first dissolved in DMSO and then diluted in phosphate-buffered saline (PBS) to a final concentration of $100 \mathrm{nM}$. We prepared fresh solutions on the day of usage. We used two stainless steel infusion cannulae (outer diameter, $300 \mu \mathrm{m}$; Muromachi-Kikai) inserted into each target region: $\mathrm{dCD}$ and $\mathrm{MDl}$ and ventroanterior thalamus and ventral putamen for controls. Each cannula was connected to a $10-\mu \mathrm{l}$ microsyringe (\#7105KH; Hamilton) via polyethylene tubing. These cannulae were advanced via guide tube by means of an oil-drive micromanipulator. DCZ or PBS was injected at a rate of $0.25 \mu \mathrm{l} / \mathrm{min}$ by auto-injector (Legato210; KD Scientific) for a total volume of $3 \mu \mathrm{l}$ for $\mathrm{dCD}$ and putamen and $2 \mu \mathrm{l}$ for $\mathrm{MDl}$ and ventroanterior thalamus for each hemisphere. For unilateral silencing, we injected either DCZ or PBS into one hemisphere. The injection volumes were determined on the basis of a previous study reporting that injection of 3 and $1.5 \mu$ of aqueous had spread about 5 to $6 \mathrm{~mm}$ and 3 to $4 \mathrm{~mm}$ in diameter in monkey brain, respectively (64). We chose enough volumes ( 3 and $2 \mu \mathrm{l}$ for $\mathrm{dCD}$ and $\mathrm{MDl}$, respectively) to cover each hM4Di-positive terminal site as we observed clear PET signals with a diameter of 5 to $7 \mathrm{~mm}$ and 3 to $4 \mathrm{~mm}$ for $\mathrm{dCD}$ and $\mathrm{MDl}$ in each hemisphere, respectively. The cannula(e) was placed at least $4 \mathrm{~mm}$ apart from the midline for both targets ( $\mathrm{dCD}$ or $\mathrm{MDl}$ ), so that the injected solution would not diffuse beyond the midline, warranting unilateral silencing of a specific pathway when injected unilaterally. The behavioral session began about $30 \mathrm{~min}$ after the infusion was finished and lasted about 1 hour.
We performed at most one silencing experiment per week for one area. At the end of each infusion, a CT image was obtained to visualize the infusion cannulae in relation to the chambers and skull. The CT image was overlaid on MR and PET images by using PMOD to verify that the infusion sites (tips of the infusion cannulae) were located in the areas in which differential PET signals were observed when radioactive DCZ was administered, indicating that the area presumably received projections from dlPFC. As a control experiment, we also injected DCZ solution into the ventroanterior, located anterior to $\mathrm{MD}$, or putamen, where discernible hM4Di expression was not observed by PET (fig. S5).

Following several unilateral infusions of DCZ into dCD, we monitored the monkeys' eye movements while their heads were fixed by using an eye-tracking camera (ETL-200, ISCAN) located $40 \mathrm{~cm}$ from the monkeys' eyes. Data were stored on a computer and then processed by custom-made software on MATLAB (MathWorks).

\section{PET imaging}

PET imaging was conducted as previously reported (35). Briefly, PET scans were conducted at 45 days after injection of vectors for both monkeys and also before injection of vectors for Mk\#2. PET scans were performed using a microPET Focus 220 scanner (Siemens Medical Solutions USA, Malvern, USA). The monkeys were immobilized by ketamine ( 5 to $10 \mathrm{mg} / \mathrm{kg}$ ) and xylazine $(0.2$ to $0.5 \mathrm{mg} / \mathrm{kg}$ ) and then maintained under anesthetized condition with isoflurane (1 to 3\%) during all PET procedures. Transmission scans were performed for about 20 min with a Ge-68 source. Emission scans were acquired in three-dimensional list mode with an energy window of 350 to $750 \mathrm{keV}$ after an intravenous bolus injection of $\left[{ }^{11} \mathrm{C}\right] \mathrm{DCZ}$ (344.8 to $369.6 \mathrm{MBq}$ ). Emission data acquisition lasted $90 \mathrm{~min}$. PET image reconstruction was performed with filtered back-projection using a Hanning filter cutoff at a Nyquist frequency of $0.5 \mathrm{~mm}^{-1}$. To estimate the specific binding of $\left[{ }^{11} \mathrm{C}\right] \mathrm{DCZ}$, regional binding potential relative to nondisplaceable radioligand $\left(\mathrm{BP}_{\mathrm{ND}}\right)$ was calculated by PMOD with an original multilinear reference tissue model using the cerebellum as a reference.

\section{Histology and immunostaining}

For histological inspection, two monkeys ( $\mathrm{Mk \# 1}$ and $\mathrm{Mk \# 3)}$ were deeply anesthetized with an overdose of sodium pentobarbital $(80 \mathrm{mg} / \mathrm{kg}$, i.v. $)$ and transcardially perfused with saline at $4^{\circ} \mathrm{C}$, followed by $4 \%$ paraformaldehyde in $0.1 \mathrm{M} \mathrm{PBS}$ ( $\mathrm{pH} 7.4$ ). The brain was removed from the skull, postfixed in the same fresh fixative overnight, saturated with $30 \%$ sucrose in $\mathrm{PB}$ at $4^{\circ} \mathrm{C}$, and then cut serially into $50-\mu \mathrm{m}$-thick sections on a freezing microtome. For visualization of immunoreactive signals of green fluorescent protein (GFP) (coexpressed with hM4Di) and $\mathrm{mKO}$ in $\mathrm{Mk} \# 1$ and $\mathrm{Mk} \# 3$, respectively, a series of every sixth section was immersed in $1 \%$ skim milk for 1 hour at room temperature and incubated overnight at $4^{\circ} \mathrm{C}$ with rabbit anti-GFP monoclonal antibody (1:500; G10362, Thermo Fisher Scientific) and rabbit anti-mKO polyclonal antibody (1:500; PM051M, Medical \& Biological Laboratories Co. Ltd., Japan), respectively, in PBS containing $0.1 \%$ Triton X-100 and $1 \%$ normal goat serum for 2 days at $4^{\circ} \mathrm{C}$. The sections were then incubated in the same fresh medium containing biotinylated goat anti-rabbit immunoglobulin $\mathrm{G}$ antibody (1:1000; Jackson ImmunoResearch, West Grove, PA, USA) for 2 hours at room temperature, followed by avidin-biotinperoxidase complex (ABC Elite, Vector Laboratories, Burlingame, CA, USA) for 2 hours at room temperature. For visualization of the 
antigen, the sections were reacted in $0.05 \mathrm{M}$ tris- $\mathrm{HCl}$ buffer ( $\mathrm{pH} 7.6)$ containing $0.04 \%$ diaminobenzidine (DAB), $0.04 \% \mathrm{NiCl}_{2}$, and $0.003 \%$ $\mathrm{H}_{2} \mathrm{O}_{2}$. The sections were mounted on gelatin-coated glass slides, air-dried, and cover-slipped. A part of other sections was Nisslstained with $1 \%$ Cresyl violet. Images of sections were digitally captured using an optical microscope equipped with a high-grade charge-coupled device camera (Biorevo, Keyence, Osaka, Japan).

\section{Statistics}

To examine the effect of each treatment on the performance of the delayed response task, behavioral measurement (correct rates) was subjected to two-way ANOVA (treatment $\times$ delay) using GraphPad Prism 7 or three-way ANOVA (treatment $\times$ delay $\times$ session) using custom-made code by Python. For the free-choice task, the choice index was calculated by the following equation

Choice index $=\left({ }^{\mathrm{ipsi}}\right.$ Choice $-{ }^{\text {contra }}$ Choice $) /\left({ }^{\mathrm{ipsi}}\right.$ Choice $+{ }^{\mathrm{contra}}$ Choice $)$

where ${ }^{\text {ipsi }}$ Choice and ${ }^{\text {contra }}$ Choice indicate the number of ipsilateral and contralateral choices, respectively, against a hemisphere in which DCZ solution was injected. Then the index was subjected to Brunner-Munzel test to compare with vehicle infusions using R.

\section{SUPPLEMENTARY MATERIALS}

Supplementary material for this article is available at http://advances.sciencemag.org/cgi/ content/full/7/26/eabg4246/DC1

View/request a protocol for this paper from Bio-protocol.

\section{REFERENCES AND NOTES}

1. S. P. Wise, Forward frontal fields: Phylogeny and fundamental function. Trends Neurosci. 31, 599-608 (2008)

2. J. M. Scimeca, D. Badre, Striatal contributions to declarative memory retrieval. Neuron $\mathbf{7 5}$, 380-392 (2012).

3. M. M. Halassa, S. Kastner, Thalamic functions in distributed cognitive control. Nat. Neurosci. 20, 1669-1679 (2017).

4. S. Parnaudeau, S. S. Bolkan, C. Kellendonk, The mediodorsal thalamus: An essential partner of the prefrontal cortex for cognition. Biol. Psychiatry 83, 648-656 (2018).

5. Y. D. Van der Werf, P. Scheltens, J. Lindeboom, M. P. Witter, H. B. Uylings, J. Jolles, Deficits of memory, executive functioning and attention following infarction in the thalamus; a study of 22 cases with localised lesions. Neuropsychologia 41, 1330-1344 (2003).

6. B. Voytek, R. T. Knight, Prefrontal cortex and basal ganglia contributions to visual working memory. Proc. Natl. Acad. Sci. U.S.A. 107, 18167-18172 (2010).

7. S. S. Bolkan, J. M. Stujenske, S. Parnaudeau, T. J. Spellman, C. Rauffenbart, A. I. Abbas, A. Z. Harris, J. A. Gordon, C. Kellendonk, Thalamic projections sustain prefrontal activity during working memory maintenance. Nat. Neurosci. 20, 987-996 (2017).

8. S. Parnaudeau, P. K. O'Neill, S. S. Bolkan, R. D. Ward, A. I. Abbas, B. L. Roth, P. D. Balsam, J. A. Gordon, C. Kellendonk, Inhibition of mediodorsal thalamus disrupts thalamofrontal connectivity and cognition. Neuron 77, 1151-1162 (2013).

9. A. S. Mitchell, J. C. Dalrymple-Alford, Dissociable memory effects after medial thalamus lesions in the rat. Eur. J. Neurosci. 22, 973-985 (2005).

10. G. Pergola, L. Danet, A.-L. Pitel, G. A. Carlesimo, S. Segobin, J. Pariente, B. Suchan, A. S. Mitchell, E. J. Barbeau, The regulatory role of the human mediodorsal thalamus. Trends Cogn. Sci. 22, 1011-1025 (2018).

11. N. D. Woodward, H. Karbasforoushan, S. Heckers, Thalamocortical dysconnectivity in schizophrenia. Am. J. Psychiatry 169, 1092-1099 (2012).

12. L. Ma, J. L. Steinberg, J. M. Bjork, L. Keyser-Marcus, J. Vassileva, M. Zhu, V. Ganapathy, Q. Wang, E. L. Boone, S. Ferre, W. K. Bickel, F. Gerard Moeller, Fronto-striatal effective connectivity of working memory in adults with cannabis use disorder. Psychiatry Res. Neuroimaging 278, 21-34 (2018).

13. D. L. Harrington, Q. Shen, J. Vincent Filoteo, I. Litvan, M. Huang, G. N. Castillo, R. R. Lee, E. Bayram, Abnormal distraction and load-specific connectivity during working memory in cognitively normal Parkinson's disease. Hum. Brain Mapp. 41, 1195-1211 (2020).

14. D. Xiao, B. Zikopoulos, H. Barbas, Laminar and modular organization of prefrontal projections to multiple thalamic nuclei. Neuroscience 161, 1067-1081 (2009).
15. N. R. McFarland, S. N. Haber, Convergent inputs from thalamic motor nuclei and frontal cortical areas to the dorsal striatum in the primate. J. Neurosci. 20, 3798-3813 (2000).

16. M. L. Schwartz, J. J. Dekker, P. S. Goldman-Rakic, Dual mode of corticothalamic synaptic termination in the mediodorsal nucleus of the rhesus monkey. J. Comp. Neurol. 309, 289-304 (1991).

17. S. M. Sherman, R. W. Guillery, The role of the thalamus in the flow of information to the cortex. Philos. Trans. R. Soc. Lond. B Biol. Sci. 357, 1695-1708 (2002).

18. S. N. Haber, The primate basal ganglia: Parallel and integrative networks. J. Chem. Neuroanat. 26, 317-330 (2003).

19. G. E. Alexander, M. R. DeLong, P. L. Strick, Parallel organization of functionally segregated circuits linking basal ganglia and cortex. Annu. Rev. Neurosci. 9, 357-381 (1986).

20. M. Kinoshita, R. Matsui, S. Kato, T. Hasegawa, H. Kasahara, K. Isa, A. Watakabe, T. Yamamori, Y. Nishimura, B. Alstermark, D. Watanabe, K. Kobayashi, T. Isa, Genetic dissection of the circuit for hand dexterity in primates. Nature 487, 235-238 (2012).

21. M. Kinoshita, R. Kato, K. Isa, K. Kobayashi, K. Kobayashi, H. Onoe, T. Isa, Dissecting the circuit for blindsight to reveal the critical role of pulvinar and superior colliculus. Nat. Commun. 10, 135 (2019).

22. T. Ninomiya, A. Noritake, K. Kobayashi, M. Isoda, A causal role for frontal cortico-cortical coordination in social action monitoring. Nat. Commun. 11, 5233 (2020)

23. P. Vancraeyenest, J. T. Arsenault, X. Li, Q. Zhu, K. Kobayashi, K. Isa, T. Isa, W. Vanduffel, Selective mesoaccumbal pathway inactivation affects motivation but not reinforcementbased learning in macaques. Neuron 108, 568-581.e6 (2020).

24. K. Inoue, M. Takada, M. Matsumoto, Neuronal and behavioural modulations by pathway-selective optogenetic stimulation of the primate oculomotor system. Nat. Commun. 6, 8378 (2015).

25. K. Maeda, K.-i. Inoue, J. Kunimatsu, M. Takada, O. Hikosaka, Primate amygdalo-nigral pathway for boosting oculomotor action in motivating situations. iScience 23, 101194 (2020).

26. H. Amita, H. F. Kim, K.-i. Inoue, M. Takada, O. Hikosaka, Optogenetic manipulation of a value-coding pathway from the primate caudate tail facilitates saccadic gaze shift. Nat. Commun. 11, 1876 (2020).

27. S. F. Owen, M. H. Liu, A. C. Kreitzer, Thermal constraints on in vivo optogenetic manipulations. Nat. Neurosci. 22, 1061-1065 (2019).

28. M. Mahn, M. Prigge, S. Ron, R. Levy, O. Yizhar, Biophysical constraints of optogenetic inhibition at presynaptic terminals. Nat. Neurosci. 19, 554-556 (2016)

29. B. L. Roth, DREADDs for Neuroscientists. Neuron 89, 683-694 (2016).

30. S. V. Mahler, E. M. Vazey, J. T. Beckley, C. R. Keistler, E. M. McGlinchey, J. Kaufling, S. P. Wilson, K. Deisseroth, J. J. Woodward, G. Aston-Jones, Designer receptors show role for ventral pallidum input to ventral tegmental area in cocaine seeking. Nat. Neurosci. 17, 577-585 (2014).

31. T. J. Stachniak, A. Ghosh, S. M. Sternson, Chemogenetic synaptic silencing of neural circuits localizes a hypothalamus $\rightarrow$ midbrain pathway for feeding behavior. Neuron $\mathbf{8 2}$, 797-808 (2014).

32. M. L. Shipman, G. C. Johnson, M. E. Bouton, J. T. Green, Chemogenetic silencing of prelimbic cortex to anterior dorsomedial striatum projection attenuates operant responding. eNeuro 6, ENEURO.0125-19.2019, (2019).

33. M. Malvaez, C. Shieh, M. D. Murphy, V. Y. Greenfield, K. M. Wassum, Distinct corticalamygdala projections drive reward value encoding and retrieval. Nat. Neurosci. 22, 762-769 (2019).

34. B. Halbout, A. T. Marshall, A. Azimi, M. Liljeholm, S. V. Mahler, K. M. Wassum, S. B. Ostlund, Mesolimbic dopamine projections mediate cue-motivated reward seeking but not reward retrieval in rats. eLife $\mathbf{8}$, e43551 (2019).

35. Y. Nagai, N. Miyakawa, H. Takuwa, Y. Hori, K. Oyama, B. Ji, M. Takahashi, X.-P. Huang, S. T. Slocum, J. F. DiBerto, Y. Xiong, T. Urushihata, T. Hirabayashi, A. Fujimoto, K. Mimura, J. G. English, J. Liu, K. Inoue, K. Kumata, C. Seki, M. Ono, M. Shimojo, M.-R. Zhang, Y. Tomita, J. Nakahara, T. Suhara, M. Takada, M. Higuchi, J. Jin, B. L. Roth, T. Minamimoto, Deschloroclozapine, a potent and selective chemogenetic actuator enables rapid neuronal and behavioral modulations in mice and monkeys. Nat. Neurosci. 23, 1157-1167 (2020).

36. P. G. F. Browning, S. Chakraborty, A. S. Mitchell, Evidence for mediodorsal thalamus and prefrontal cortex interactions during cognition in macaques. Cereb. Cortex 25, 4519-4534 (2015).

37. O. Hikosaka, M. Sakamoto, S. Usui, Functional properties of monkey caudate neurons. II. Visual and auditory responses. J. Neurophysiol. 61, 799-813 (1989).

38. M. Kato, N. Miyashita, O. Hikosaka, M. Matsumura, S. Usui, A. Kori, Eye movements in monkeys with local dopamine depletion in the caudate nucleus. I. Deficits in spontaneous saccades. J. Neurosci. 15, 912-927 (1995).

39. P. S. Goldman-Rakic, L. J. Porrino, The primate mediodorsal (MD) nucleus and its projection to the frontal lobe. J. Comp. Neurol. 242, 535-560 (1985).

40. M. Giguere, P. S. Goldman-Rakic, Mediodorsal nucleus: areal, laminar, and tangential distribution of afferents and efferents in the frontal lobe of rhesus monkeys. J. Comp. Neurol. 277, 195-213 (1988). 
41. E. H. Yeterian, D. N. Pandya, Laminar origin of striatal and thalamic projections of the prefrontal cortex in rhesus monkeys. Exp. Brain Res. 99, 383-398 (1994).

42. G. D. Horwitz, W. T. Newsome, Separate signals for target selection and movement specification in the superior colliculus. Science 284, 1158-1161 (1999).

43. J. M. Fuster, The Prefrontal Cortex (Academic Press, Boston, 2015).

44. P. S. Goldman-Rakic, Cellular basis of working memory. Neuron 14, 477-485 (1995).

45. S. Funahashi, Thalamic mediodorsal nucleus and its participation in spatial working memory processes: Comparison with the prefrontal cortex. Front. Syst. Neurosci. 7, 36 (2013).

46. N. Neave, A. Sahgal, J. P. Aggleton, Lack of effect of dorsomedial thalamic lesions on automated tests of spatial memory in the rat. Behav. Brain Res. 55, 39-49 (1993).

47. A. S. Mitchell, P. G. F. Browning, M. G. Baxter, Neurotoxic lesions of the medial mediodorsal nucleus of the thalamus disrupt reinforcer devaluation effects in rhesus monkeys. J. Neurosci. 27, 11289-11295 (2007).

48. L. I. Schmitt, R. D. Wimmer, M. Nakajima, M. Happ, S. Mofakham, M. M. Halassa, Thalamic amplification of cortical connectivity sustains attentional control. Nature 545, 219-223 (2017).

49. F. Alcaraz, V. Fresno, A. R. Marchand, E. J. Kremer, E. Coutureau, M. Wolff, Thalamocortical and corticothalamic pathways differentially contribute to goal-directed behaviors in the rat. elife 7, e32517 (2018).

50. T. Sawaguchi, M. Iba, Prefrontal cortical representation of visuospatial working memory in monkeys examined by local inactivation with muscimol. J. Neurophysiol. 86, 2041-2053 (2001).

51. Y. Watanabe, S. Funahashi, Neuronal activity throughout the primate mediodorsal nucleus of the thalamus during oculomotor delayed-responses. I. Cue-, delay-, and response-period activity. J. Neurophysiol. 92, 1738-1755 (2004).

52. K.-I. Tsutsui, F. Grabenhorst, S. Kobayashi, W. Schultz, A dynamic code for economic object valuation in prefrontal cortex neurons. Nat. Commun. 7, 12554 (2016).

53. C. F. Jacobsen, Studies of cerebral function in primates. I. The functions of the frontal association areas in monkeys. Comp. Psychol. Monogr. 13, 1-60 (1936).

54. T. E. Hazy, M. J. Frank, R. C. O'Reilly, Towards an executive without a homunculus: Computational models of the prefrontal cortex/basal ganglia system. Philos. Trans. R. Soc. Lond. B Biol. Sci. 362, 1601-1613 (2007).

55. C. H. Chatham, D. Badre, Multiple gates on working memory. Curr. Opin. Behav. Sci. 1, 23-31 (2015).

56. L. S. Geiger, C. Moessnang, A. Schäfer, Z. Zang, M. Zangl, H. Cao, T. R. van Raalten, A. Meyer-Lindenberg, H. Tost, Novelty modulates human striatal activation and prefrontal-striatal effective connectivity during working memory encoding. Brain Struct. Funct. 223, 3121-3132 (2018).

57. P. Apicella, E. Legallet, A. Nieoullon, E. Trouche, Neglect of contralateral visual stimul in monkeys with unilateral striatal dopamine depletion. Behav. Brain Res. 46, 187-195 (1991).
58. K. Johnston, S. G. Lomber, S. Everling, Unilateral deactivation of macaque dorsolateral prefrontal cortex induces biases in stimulus selection. J. Neurophysiol. 115, 1468-1476 (2016).

59. K. Samejima, Y. Ueda, K. Doya, M. Kimura, Representation of action-specific reward values in the striatum. Science 310, 1337-1340 (2005).

60. R. Kawagoe, Y. Takikawa, O. Hikosaka, Expectation of reward modulates cognitive signals in the basal ganglia. Nat. Neurosci. 1, 411-416 (1998).

61. A. S. Mitchell, The mediodorsal thalamus as a higher order thalamic relay nucleus important for learning and decision-making. Neurosci. Biobehav. Rev. 54, 76-88 (2015).

62. K. Deisseroth, Optogenetics. Nat. Methods 8, 26-29 (2011).

63. N. A. Upright, M. G. Baxter, Effect of chemogenetic actuator drugs on prefrontal cortex-dependent working memory in nonhuman primates. Neuropsychopharmacology 45, 1793-1798 (2020)

64. Y. Murata, N. Higo, T. Hayashi, Y. Nishimura, Y. Sugiyama, T. Oishi, H. Tsukada, T. Isa, $\mathrm{H}$. Onoe, Temporal plasticity involved in recovery from manual dexterity deficit after motor cortex lesion in macaque monkeys. J. Neurosci. 35, 84-95 (2015).

Acknowledgments: We thank J. Kamei, R. Yamaguchi, Y. Matsuda, Y. Sugii, T. Okauchi, R. Suma, A. Maruyama, T. Kokufuta, Y. Iwasawa, T. Watanabe, A. Tanizawa, S. Shibata, N. Nitta, Y. Ozawa, M. Fujiwara, and M. Nakano for the technical assistance. Funding: This study was supported by MEXT/JSPS KAKENHI grant numbers JP18K15353 and JP21K07268 (to K.O.), JP17H02219 (to T.H.), JP19H05467 (to M.T.), JP15H05917 and JP18H04037 (to T.M.), and JP18H05018, JP19K07811, and QST President's Strategic Grant (Creative Research) (to N.M.); by AMED grant numbers JP20dm0307007 (to T.H.), JP20dm0307021 (to K.I.), JP18dm0207003 (to M.T.), and JP20dm0107146 (to T.M.); by JST PRESTO Grant Number JPMJPR1683 (to K.I.); by the cooperative research program at PRI, Kyoto Univ.; and by National Bio-Resource Project "Japanese Monkeys" of MEXT, Japan. Author contributions: Conceptualization: T.M. investigation: K.O., Y.H., and Y.N.; resources: K.I. and M.T.; visualization: K.O.; Writing-review and editing: All authors. Competing interests: The authors declare that they have no competing interests. Data and materials availability: All data needed to evaluate the conclusions in the paper are available at https://github.com/minamimoto-lab/2021-OyamaDLPFC. Additional data related to this paper may be requested from the authors.

Submitted 6 January 2021

Accepted 10 May 2021

Published 23 June 2021

$10.1126 /$ sciadv.abg4246

Citation: K. Oyama, Y. Hori, Y. Nagai, N. Miyakawa, K. Mimura, T. Hirabayashi, K.-i. Inoue, T. Suhara, M. Takada, M. Higuchi, T. Minamimoto, Chemogenetic dissection of the primate prefronto-subcortical pathways for working memory and decision-making. Sci. Adv. 7 , eabg4246 (2021). 


\section{ScienceAdvances}

\section{Chemogenetic dissection of the primate prefronto-subcortical pathways for working memory and decision-making}

Kei Oyama, Yukiko Hori, Yuji Nagai, Naohisa Miyakawa, Koki Mimura, Toshiyuki Hirabayashi, Ken-ichi Inoue, Tetsuya Suhara, Masahiko Takada, Makoto Higuchi and Takafumi Minamimoto

Sci Adv 7 (26), eabg4246.

DOI: $10.1126 /$ sciadv.abg4246

ARTICLE TOOLS

SUPPLEMENTARY

MATERIALS

REFERENCES

PERMISSIONS http://advances.sciencemag.org/content/7/26/eabg4246

http://advances.sciencemag.org/content/suppl/2021/06/21/7.26.eabg4246.DC1

This article cites 62 articles, 7 of which you can access for free http://advances.sciencemag.org/content/7/26/eabg4246\#BIBL

http://www.sciencemag.org/help/reprints-and-permissions

Science Advances (ISSN 2375-2548) is published by the American Association for the Advancement of Science, 1200 New York Avenue NW, Washington, DC 20005. The title Science Advances is a registered trademark of AAAS.

Copyright @ 2021 The Authors, some rights reserved; exclusive licensee American Association for the Advancement of Science. No claim to original U.S. Government Works. Distributed under a Creative Commons Attribution NonCommercial License 4.0 (CC BY-NC). 